\title{
Tensiones disciplinales entre la Contabilidad Financiera y la Tributación producidas por su lenguaje especializado
}

\author{
Bastidas Canelones, Jorge* \\ https://orcid.org/0000-0002-5076-0249 \\ *Universidad de los Andes, Mérida, Venezuela \\ E-mail: jorgebastidas1983@gmail.com
}

Recibido: 10 de enero de 2021

Aprobado: 22 de enero de 2021

\section{Resumen}

En la primera entrega de esta serie de reflexiones, se intentó demostrar el carácter interdisciplinario entre la Contabilidad Financiera y la Tributación, como preámbulo a develar las tensiones que se producen en la relación diaria y práctica entre estos dos conocimientos. En esta oportunidad, se intentará reflexionar brevemente sobre el lenguaje especializado utilizado en ambas disciplinas, y como resultado de esta reflexión se pudiera afirmar que posiblemente el alto grado de especialización en el lenguaje de ambas disciplinas produce tensiones en la interacción que se da entre ambos conocimientos, y que, pudieran afectar la práctica profesional diaria del Contador Público. El alto grado de especialización se debe a que las estructuras semánticas y semióticas presentes en sus discursos, provienen, posiblemente, del sistema de creencia disímil presente en el interior de cada disciplina. No obstante, el sistema de creencia no será analizado sino hasta el final de esta serie de reflexiones.

Palabras clave: Lenguaje, Contabilidad Financiera, Tributación, Creencia. 


\begin{abstract}
In the first volume of this series of reflections, an attempt was made to demonstrate the interdisciplinary nature between Financial Accounting and Taxation, as a preamble to uncovering the tensions that occur in the daily and practical relationship between these two types of knowledge. On this occasion, an attempt will be made to briefly reflect on the specialized language used in both disciplines, and as a result of this reflection, it could be stated that possibly the high degree of specialization in the language of both disciplines produces tensions in the interaction that occurs between both knowledge, and probably can affect the daily professional practice of the Public Accountant. The high degree of specialization is due to the fact that their semantic and semiotic structures present in their discourses come, possibly, from the dissimilar belief system present within each discipline. However, the belief system will not be analyzed until the end of this series of reflections.
\end{abstract}

Keywords: Language, financial accounting, taxation, belief

\section{Introducción}

El lenguaje contable y tributario es producto de "construcciones sociales que dependen de la intencionalidad del sujeto emisor" Pacheco (2013 p. $65)$, es decir, la intención que tenga el ente regulador de las Normas Internacionales de Información Financiera (IASB por sus siglas en inglés), incidirá en la configuración de su lenguaje y, el Poder Legislativo local, tendrá su intención propia produciendo también su propio lenguaje. Estos lenguajes representan a un sistema de creencias propio de esas instituciones que a la postre formará parte de un discurso diseñado para imponer o persuadir sobre aspectos de la empírea económica a los practicantes de una disciplina. A continuación, la metodología utilizada para la presente investigación que soporta los hallazgos encontrados.

\section{Metodología de revisión bibliográfica}

En cuanto a la metodología usada para la revisión bibliográfica se procedió a revisar sistemáticamente una serie de documentos de sociedades científicas dedicadas a la investigación en el área contable y tributaria, así como la posición de la doctrina sobre la construcción de los lenguajes que tienen cada una de ellas. Se incluyó literatura y, mediante una revisión bibliográfica empírica, se intentó hallar la diferencia en los lenguajes presentes en los discursos disciplinarios tanto de la Tributación como de la Contabilidad Financiera que permitan visualizar la forma en que se comunican estas disciplinas.
En cuanto a la estrategia de búsqueda se procedió a revisar en revistas científicas (Accounting, Organizations and Society, American Accounting, The Accounting View, Actualidad Contable, Vision Contable, etc.) y en bibliotecas digitales tales como Redalyc, Scielo, Dialnet, Saberula entre otras, de artículos científicos relacionados con el área objeto de estudio. Esta búsqueda se hizo tanto en español, como en inglés, italiano y portugués, sin límite de fecha. Se analizaron además las referencias bibliográficas de los artículos seleccionados en inglés con el fin de determinar estudios potencialmente incluibles para la presente revisión.

En la búsqueda de literatura gris se incluyó además documentos como sentencias en original, leyes tributarias y constituciones de algunos países de Latinoamérica. Respecto a las revisiones de los estudios científicos, el criterio de inclusión que se usó fue el que los estudios incluyeran posiciones sobre las prácticas disciplinales derivadas de un marco de referencia (constituciones, leyes, NIIF) y sobre las visiones que tienen los autores sobre las formas en como se construyen esos lenguajes.

Para proceder a la selección se revisaron los títulos, los resúmenes y las conclusiones para decidir si era necesario leer los argumentos esgrimidos en los artículos que suministrarán aportes importantes a la presente investigación.

Sin embargo, es de destacar que en esta investigación se privilegia el accionar del propio 
investigador y el contexto tributario en el cual se desenvuelve, planteando críticas, vacíos y nuevas formas de visualizar la disciplina tributaria dado que la postura del investigador es crítico interpretativa.

En el cuadro 1 se presenta el formato de registro de observación documental utilizado para la realización del presente estudio. En este se detalla el propósito general del estudio las consecuencias, las categorías a documentar y las observaciones que hiciere el investigador sobre el tópico investigado. Para efectos de este artículo se omitieron las observaciones pues representan las anotaciones a primera vista que hace el investigador sobre los documentos revisados y descartados.

Tabla 1

Registro de Observación Documental

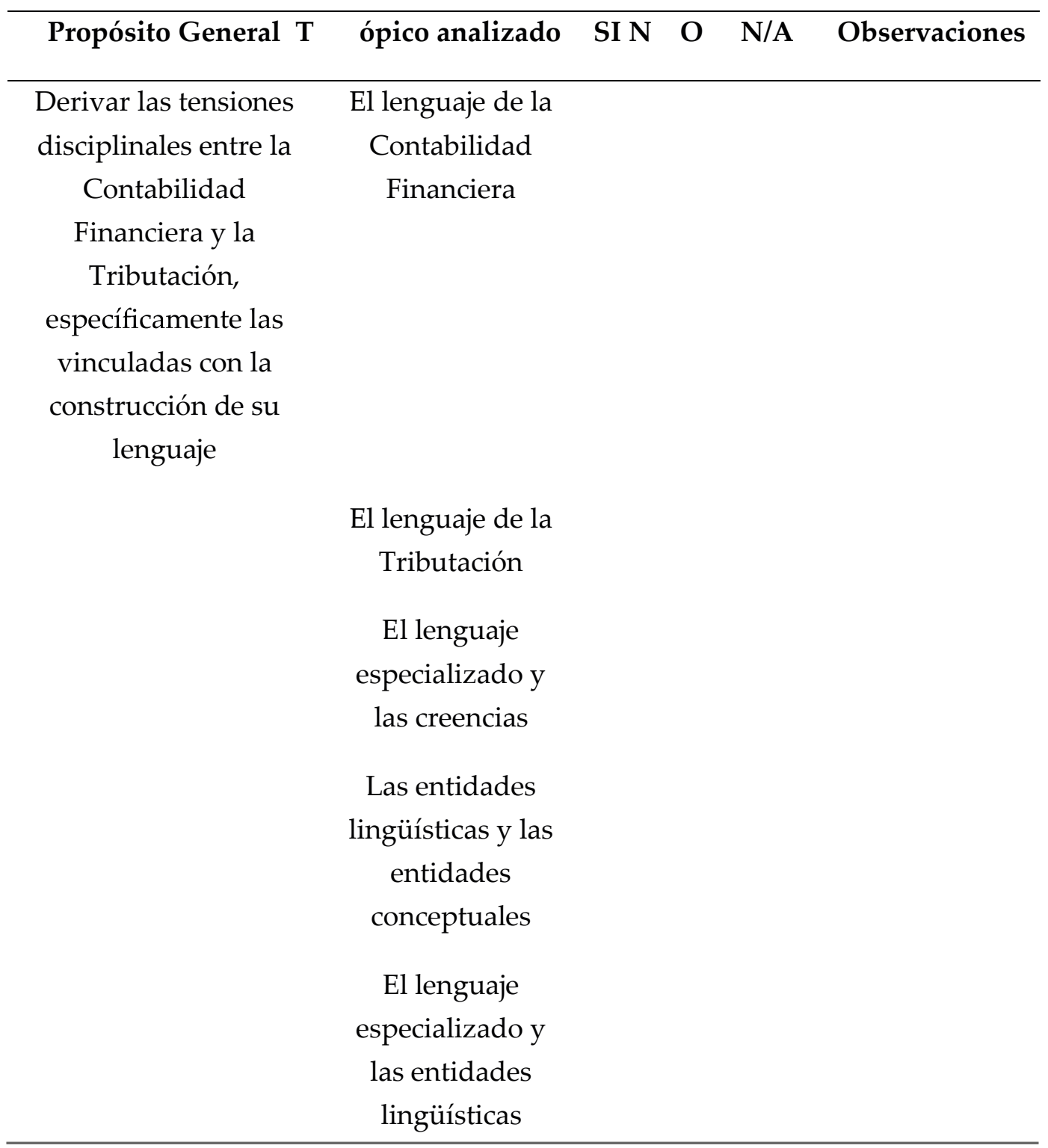

Fuente: Elaboración Propia 2020 


\section{El lenguaje tributario}

La Contabilidad Financiera y la Tributación cuentan con su propio lenguaje especializado para representar la realidad. El lenguaje de la Tributación evidencia un carácter meramente prescriptivo, sancionatorio $y$ con un fuerte componente cuantitativo. $\mathrm{Su}$ origen obedece a la necesidad de recaudar fondos para el financiamiento de un Estado por lo que se alimenta primariamente del lenguaje del Derecho y de la Economía, a diferencia de la Contabilidad Financiera que se alimenta primariamente de las finanzas.

A pesar que la Tributación se alimenta, tanto del lenguaje de otras disciplinas, como del lenguaje común, en su intención de regular las conductas de las personas naturales o jurídicas en determinados contextos impositivos, también ha desarrollado su propio lenguaje jurídico y económico a través de la modulación y absorción de la nomenclatura y semántica del lenguaje especializado de esos conocimientos, entre los que se destaca la Contabilidad. Con este procedimiento de "adopción y adaptación se desarrollan nuevas acepciones y conceptos de aplicación propia" (Senado Mexicano 2010 p. 6) para esta área del conocimiento. La tensión se produce cuando este lenguaje tributario entre en contacto con otros lenguajes especializados en la interacción disciplinal y no considera las formas comunicativas y signos lingüísticos de otros conocimientos.

Esto pudiera ser llevado al plano contable ya que en su absorción semántica de otros conocimientos yace la consideración de conceptos como Activos, Pasivos, Ingresos, Costos y Gastos que provienen de la contabilidad y no de los confines de su propia disciplina, esto pudiera traer deformaciones en los instrumentos jurídicos que regulen las actividades económicas e impositivas de un país ya que se adapta estos conceptos a las necesidades recaudatorias y temporales del Estado y no a los avances doctrinarios y paradigmáticos de la disciplina contable, o a la de cualquier otra disciplina considerada para fines regulativos.

\subsection{EI lenguaje de la Contabilidad Financiera}

Por su parte, el lenguaje de la Contabilidad Financiera presenta una mezcla entre la Contabilidad y las Finanzas por lo que el mercado (y no la empírea económica) incide de forma directa en lo que este tipo de Contabilidad especializada intenta representar. Se recordar que la economía y las finanzas son disciplinas diferentes. En las finanzas se considera primero el mercado y el valor del dinero en el tiempo como forma de medición de hechos y transacciones que se dieron en la empírea antes que los precios de intercambio y los referentes empíricos que sí considera la economía. En la revisión bibliográfica se evidencian sintagmas vinculados al valor presente de los flujos, al valor de uso, al valor razonable, a los tipos financieros para descontar los fondos, etc.

A diferencia de la Contabilidad que relata hechos del pasado, la Contabilidad Financiera comunica sobre los hechos y transacciones que se dieron y también los que pudieran producirse; es predictiva por naturaleza y así lo evidencia su lenguaje tal y como se mostró en el artículo anterior en esta misma revista (Ver posturas del autor sobre "la Razonabilidad de la Información Financiera", Revista Sigma 2020) lo que causa evidentes consecuencias en su interacción con la Tributación ya que la tributación es histórico, le interesa hechos acaecidos que pudieran dar origen a la obligación tributaria material, no le interesa los hechos que acaecerán.

En opinión del investigador, la especialización en los lenguajes tributarios y contables se produce a partir de la suma agregada de creencias que son validadas o desechadas continuamente en los confines de esas disciplinas, lo que hace que producto de la evolución de los negocios y del contexto social, haya cambios en las creencias y por consiguiente, en los significantes y significados que se derivan a partir de la representación de esas creencias. Y es que estos lenguajes especializados "tienen creencias propias que fungen como su soporte cognitivo" (Van Dijk 2016 p. 3598), lo que pudiera potenciar, no solo la polisemia en los discursos propios, sino las tensiones discursivas cuando estas disciplinas entren 
en contacto con otras áreas del conocimiento. Como ejemplo de polisemia, Machado (2016) señala que la polisemia produce tensiones discursivas que no solo se producen entre disciplinas que interactúan entre sí, sino al interior de cada disciplina. Un ejemplo de esta situación es la consideración de

(...) un concepto contable tan importante como el de Activo, el cual acusa discrepancia entre los organismos emisores y los académicos por su definición. Tal es el caso de International Accounting Standards Board (IASB), que en su Marco Conceptual del 2010 (párrafo 4.4, p. 18) lo define como "un recurso controlado por la entidad como resultado de sucesos pasados, del que la entidad espera obtener, en el futuro, beneficios económicos" mientras que académicos como Kieso y Weygandt (1999, p. 40) lo definen como "probables beneficios económicos futuros obtenidos o controlados por una entidad en particular, como resultado de transacciones o acontecimientos anteriores"; ha de observarse que mientras los primeros definen el activo como un "recurso controlado" es decir como el derecho exclusivo sobre el uso y el usufructo del mismo, los segundos lo definen como "beneficios económicos futuros" (se tenga o no control sobre el recurso) o sea como la capacidad de generar flujos de caja netos con dichos recursos, difiriendo en el significado que le atribuyen al concepto de "activo" (p. 16)

El concepto de Activo cambia ligeramente para la versión 2018 del Marco Conceptual para la Preparación de Información Financiera, definiéndolo como "un recurso económico presente" controlado por la entidad y aclarando que un recurso económico "es un derecho que tiene el potencial de producir beneficios económicos" (párrafo 4.2, p. 28) Este concepto dista mucho de lo que desde el mundo tributario se concibe como Activo, ya que ante la ausencia de un concepto propio de Activo desarrollado en los confines de esa disciplina, en ocasiones, en la práctica se obvia el concepto contable del "control" para categorizar a un bien o derecho como un Activo, pero se privilegia el concepto de propiedad o tutela jurídica del bien.
Lo que sí es evidente es que cada disciplina tiene conceptos diferentes con relación a áreas u objetos de estudio en común y que probablemente los soportes cognitivos de esos conceptos también lo sean.

En estos discursos contables, tal y como lo manifiesta Machado (2016), se manifiestan "vacíos, contradicciones, borrosidades, falta de consideración de los contextos, indefiniciones y otros aspectos relacionados con el tratamiento de los conceptos generales de cada disciplina" (p. 16), y que en particular hacen que sus discursos parezcan confusos y con poco aporte al desarrollo de la interdisciplinariedad.

\subsection{EI lenguaje especializado y las creencias}

Por otro lado, no solo el lenguaje especializado que existe en la Contabilidad Financiera y la Tributación puede producir tensiones comunicativas con otras disciplinas. Con el ejemplo mencionado sobre el concepto de Activo, se demostró cómo un término puede tener diferentes connotaciones y éstas pueden obedecer, entre otras cosas, a ideologías y creencias inmanentes en la disciplina contable y tributaria. Como se mencionó, en el proceso de significación - designación que se hace en la sociedad, son muchas las comunidades epistémicas que participan las cuales orbitan en contextos y situaciones comunicativas diferentes, lo que hace que se multipliquen las formas de recrear y entender el mundo.

Es de destacas que las creencias se transforman en conocimiento a "partir de la relación sintagmática constante entre conceptos" (Van Dijk 2017 p. 10539) para luego ser validadas por los criterios de las comunidades epistémicas, es decir, las creencias se construyen por las relaciones múltiples de conceptos que van constituyendo unidades sintácticas, lo que le da nuevas características especializadas al lenguaje. Estas características son validadas continuamente por las respectivas comunidades epistémicas.

Las creencias, a menudo, "están fuertemente relacionadas con ideologías y también son controladas por las restricciones del contexto" $(\mathrm{Ob}$. 
Cit.) Estas permiten darle coherencia al discurso que se produce/interpreta, pero, al ser incorporadas a este, hacen que se pueblen de múltiples visiones de la realidad. Un discurso no puede ser producido/ interpretado sin un sistema de creencias presente en los actores sociales que participan en una acción comunicativa.

Si los discursos tributarios y contables tienen un grado de incompatibilidad en cuanto a las creencias que los soportan, pareciera obvio pensar que eso ocurra en niveles estructurales del discurso como en las estructuras semánticas o semióticas en vista de que el lenguaje también representa un sistema de creencias. Ello puede explicar el hecho de que ambas disciplinas denoten a un mismo hecho económico de manera diferente; utilicen un mismo significante para representar hechos o conceptos diferentes y no reconozcan hechos que son de necesaria aplicación para una u otra disciplina en la praxis, lo que potencie, probablemente, las tensiones y controversias en los discursos que afectan la comunicación entre ambos conocimientos.

En conclusión, a mayor diversidad de creencias que soportan el lenguaje presente en los discursos disciplinales, mayor la posibilidad que existan tensiones discursivas tanto, al interior de cada disciplina, como en las interacciones con otros conocimientos afines.

Si se dan estos argumentos por válidos, es posible afirmar tres cosas: 1) El contexto de interacción disciplinal y la variedad de creencias inmanentes en las disciplinas inciden en la construcción del lenguaje especializado presente en los discursos; 2) A mayor grado de especialización semiótica, semántica, sintagmática y sintáctica de la disciplina, sobre todo en las ciencias sociales, menor probabilidad de generar vasos comunicantes efectivos con disciplinas afines; y 3) Las creencias subyacentes en los conceptos disciplinales pudieran explicar las tensiones en los discursos, bien sea al interior de la disciplina, o con otros discursos de disciplinas afines con las que les toque interactuar.

\subsection{Las entidades lingüísticas y las entidades conceptuales}

Tampoco pudiera descartarse que tal vez algunas entidades conceptuales, tanto en la Contabilidad Financiera como en la Tributación, sean expresadas incorrectamente por las entidades lingüísticas o simplemente no sean reconocidas. Según Maldonado (1987) las entidades lingüísticas comprenden todas "aquellas que conforman el sistema de símbolos que forman el lenguaje, junto con sus correspondientes propiedades sintácticas, semánticas y fonológicas" (p. 18) y las entidades conceptuales son aquellas representaciones mentales de objetos, constructos, construcciones teóricas de hechos o fenómenos de la realidad.

En este orden, la relación entre el nivel lingüístico y el nivel conceptual se denomina relación de designación (Bunge 1967) Es decir, las entidades lingüísticas "designan las entidades conceptuales o lo que es lo mismo, las entidades conceptuales se expresan por medio de las lingüísticas. La entidad conceptual que es objeto de designación se denomina designatum y las expresiones que la designan, se llaman designans." (Maldonado 1987, Ob. Cit) Cuando la relación es inversa, que va desde lo conceptual hacia lo lingüístico, la relación es de expresión.

Para el caso del no reconocimiento de entidades conceptuales de índole contable por parte de la Tributación, Bastidas (2017) afirma que las leyes tributarias venezolanas no contemplan conceptos tales como:

El interés implícito, las provisiones, los valores netos de realización, valores de uso, precios estimados de venta, costos de desmantelamiento, el deterioro acumulado, las operaciones conjuntas, el valor presente, la transferencia de riesgos y ventajas, los pasivos y activos contingentes, los costos de instalación, los métodos para la valoración de instrumentos financieros, el concepto de impuesto diferido, entre otros, ni tampoco contempla la diferencia entre los conceptos de "gasto por impuesto sobre la renta" e "impuesto sobre la renta por pagar (p. 178)

Del mismo modo, para el caso de conceptos 
expresados incorrectamente por las entidades lingüísticas puede considerarse el siguiente ejemplo sobre la significación del "Ingreso" que debe hacer la Tributación. Al momento de significar el término "Ingreso", los productores del discurso tributario deben considerar no solo que ella no es la disciplina idónea para tal finy, si se acude a otros conocimientos, como cuando acude a la Contabilidad Financiera para efectos impositivos, debe entender que la misma prima los valores financieros por sobre los económicos, como es el caso del diferimiento en el cobro de un ingreso, cuyo valor razonable a la fecha en que se reporta es el valor presente de los cobros futuros de esa venta (IASB PYME Sección 23) y no los precios de intercambio. Por consiguiente, la tributación tendrá diversidad de valores a los fines de la determinación de algún impuesto que considere al ingreso contable como Base Imponible.

Además, también debería ver lo siguiente: Si contempla un concepto de "Ingreso" para fines tributarios distinto a los que se contemplan para la Contabilidad Financiera, no podrá acudir a ella como referente probatorio de una determinada transacción, pues probablemente la misma no sea representada por esta disciplina o la represente de forma distinta. Una solución para esta situación es el desarrollo de conceptos interdisciplinarios que permitan que ambas disciplinas denoten y signifiquen la empírea económica de forma similar cuando la praxis así lo requiera.

$\mathrm{Si}$ bien es cierto que el lenguaje como construcción social es arbitrario, también es cierto que sus signos lingüísticos cambian. En este contexto de cambios, Mires (2018) afirma que "no es el significado lo que crea al significante sino el significante al significado. Poder tan poderoso que obliga ajustar constantemente al significante con lo que desea significar, pues estos crean realidades" (p. 3)

Esto lo preveía Sasurre (1913) cuando acuñaba el enunciado "mutabilidad del signo lingüístico" en un plano diacrónico. En otras palabras, lo que les da existencia a las entidades conceptuales son las entidades lingüísticas, pero, en un plano temporal, es decir, son válidas para un momento específico en el tiempo. No obstante, a juicio del investigador, para que los procesos de designación de las entidades conceptuales estén acorde con la razón debe existir una congruencia entre: 1) El periodo histórico en el que se usa ese lenguaje, el contexto de interacción social en el que se usa, el significante y sus usos en el lenguaje ordinario, el significante y sus usos en el lenguaje especializado; y 2) Las entidades conceptuales.

Esta idea la sostuvo primariamente Sasurre (1913) al sostener que un "sistema de símbolos, el símbolo guarda una relación racional y recíproca con la cosa significada" (p. 96) pero no consideraba el contexto de la interacción comunicativa como variable de esa relación entre significado y significante. Por ejemplo, en Argentina y Brasil la palabra "quilombo" significaba a las organizaciones sociales de personas que se emancipan de la esclavitud. Luego, en Argentina la palabra designaba un prostíbulo usado por las clases menos pudientes en el siglo XIX y en las que trabajaban personas afrodescendientes, pero, el significado de la palabra transmutó y hoy designa a un "problema o lío" ante la desaparición de esos espacios sociales. Probablemente, ahora signifique "lío" por los problemas que representaban para una persona si era descubierto en alguno de esos sitios. Lo que sí es evidente es que las palabras guardan una relación con el contexto histórico en el que se usan.

Producto, entre otras cosas, de la arbitrariedad del lenguaje y del uso que le de la sociedad y sus convenciones, se producirán las mutaciones en los signos lingüísticos tanto en el lenguaje común como en el especializado. En Contabilidad, las convenciones sociales sostuvieron que la palabra "Ingreso" designaba aumentos de patrimonio medido a precios de intercambio y hoy designan aumentos de patrimonio que pueden estar medidos tanto a valores históricos como a valores futuros. La contabilidad no está exenta de este fenómeno, pero dado el carácter normativista de la disciplina, parecieran que la intencionalidad del ente emisor es la que define lo que se significa de la empírea económica y la forma de denotarlo. Esa intencionalidad esta obviamente incardinada en un sistema de creencias. 
Si los practicantes de las disciplinas no consideran que las entidades lingüísticas deben ser congruentes con las entidades conceptuales que intentan denotar harán mella en sus vasos comunicantes. En este orden, por ejemplo, si la definición de "Ingresos" del Marco Conceptual (2018) sostiene que son "aumentos en los activos, o disminuciones en los pasivos, que resultan en aumentos en el patrimonio" (párrafo 4.2), la Contabilidad Financiera debe ser cónsona con el significante, ergo, tanto el nombre de la cuenta que refleje ese aumento en el patrimonio, como la forma en que se mide ese incremento, debe ser congruente con la entidad conceptual.

En el lenguaje contable los significantes no son solo palabras (cuentas), palabras articuladas con otras (notas a los estados financieros) sino también números (Estados Financieros) Al explanar el concepto de "Ingreso" se contempla el enunciado "aumentos de patrimonio", no "posibles aumentos" y aumento para el lenguaje ordinario significa que la cantidad de una cosa es mayor luego de un evento. Entonces, si la cosa es mayor, debe existir certeza de cuanto era y de cuanto es la cosa para poder determinar en cuánto fue el incremento de la misma, es decir, no se puede afirmar que hubo un aumento de la cosa sin saber la medida exacta del incremento, pues al comparar ambos momentos, se sabe con certeza cuánto había antes y después del evento que produce el aumento.

Del mismo modo, enuncia las palabras "resultan en", no "probablemente resultarán en" por lo que mal podría el productor de normas contables establecer para las mediciones del "Ingreso" criterios con un alto componente predictivo. Lo congruente es privilegiar los precios de intercambio y no incorporar los valores futuros o de mercado como forma de expresión lingüística de ese fenómeno, ya que, al enunciar un "aumento del patrimonio," no solo debe existir la certeza de que efectivamente se produjo, sino también la medida exacta de ese aumento.

De haber sostenido que el "Ingreso" se definía como "posibles aumentos patrimoniales", el sintagma (significante) más adecuado pudiera haber sido "Ingresos Ordinarios Probables". No obstante, es sabido el carácter teleológico del modelo de regulación contable internacional y este planteamiento es poco probable que se materialice ya que, como se describió, la información es útil a los usuarios a partir de que se pueda estimar los posibles flujos futuros de recursos y de que puedan afectar sus decisiones en cuanto a la obtención de recursos para la entidad.

En este contexto, también cabe hacer mención a las erogaciones de dinero. Una erogación puede ser reconocida en la Contabilidad Financiera con un símbolo llamado Costo, o Gasto, o Activo, sin embargo, para la Tributación, estos significantes pueden tener otro significado distinto al que tiene para la Contabilidad Financiera. Un ejemplo emblemático se visualiza con el concepto de "arrendamiento".

Para la Contabilidad Financiera (IASB PYME, 2015) este concepto representa un acuerdo por el que el arrendador cede al arrendatario, a cambio de percibir una suma única de dinero, o una serie de pagos o cuotas, el derecho a utilizar un activo durante un periodo determinado $\mathrm{y}$, dependiendo si hay o no opción a compra al final del contrato, se categorizará como Financiero u Operativo. Este acuerdo debe ser reconocido en contabilidad como un Activo pues cumple con los criterios de designación que se originan a partir de la definición de Activo establecida en el Marco Conceptual (2018).

Sin embargo, para la Tributación, específicamente la Ley de Impuesto sobre la Renta venezolana (2015), este término representa un gasto deducible de los ingresos obtenidos en un ejercicio fiscal a los fines de determinar el Enriquecimiento Neto Gravable en ese periodo (Art. 27 literal 12) Los cánones o cuotas correspondientes al arrendamiento de bienes destinados a la producción de la renta no están asociados al valor de un Activo para la Tributación venezolana, pues de representarlo, hubieran sido incorporado en la ley en el Capítulo II que estipula los Costos que serán imputables y no en el Capítulo III de los Gastos que serán deducibles.

Esto también genera un vacío jurídico sobre qué hacer con la depreciación del Activo utilizado bajo la figura de arrendamiento financiero en cuanto a 
su deducción o no de los ingresos gravables, pues generalmente este tipo de Activos son utilizados en la producción de la renta y cumpliría con los criterios del articulo 27 literal 12. Hay criterios encontrados de la doctrina sobre este particular, pues un sector afirma que al deducir la depreciación de los ingresos gravables se estaría afectando doblemente la base imponible ${ }^{1}$.

En resumen, para la Tributación venezolana estos hechos deben ser reconocidos como un gasto deducible y para la Contabilidad Financiera este tipo de hechos son designados como un Activo pues son un recurso controlado, con beneficios económicos futuros y cuya depreciación es perfectamente aprovechable en atención a la forma en como fluyan los beneficios de económicos de ese bien en el futuro (IASB PYME Sección 20 párrafo 12 y Sección 17)

Tal como se ha mencionado, las tensiones discursivas producto de la estructuración de los lenguajes contables y tributarios son muy evidentes, por lo que cabe preguntarse si:

a) ¿El lenguaje especializado utilizado por ambas disciplinas en esa interacción debe ser compatible?

b) ¿Las creencias subyacentes que definen los conceptos en cada disciplina deben ser compatibles?

c) ¿Cuáles son las condiciones que deben existir para que en esa interacción disciplinal se garantice el objetivo de cada disciplina?

d) ¿Qué características debe tener el discurso de ambas disciplinas que garanticen la comunicación interdisciplinaria?

Sin entrar a responder estas interrogantes, las mismas surgen porque la interacción rutinaria entre la Contabilidad Financiera y la Tributación hace pensar que probablemente el discurso que permita a ambas disciplinas comunicarse debe ser compatible, de lo contrario, se dificultará el proceso efectivo de intercambio de datos y aplicación conjunta de normativas contables y tributarias y, por lo tanto, traería consecuencias en la praxis disciplinal.

Ferullo et al (2006) destacan la existencia de un grado de compatibilidad necesaria entre el discurso tributario y el contable que permita a ambas disciplinas el cumplimiento de sus fines, al realzar la importancia de una comunicación efectiva entre ambas disciplinas, motivado, a las zonas de dependencia pragmática que existen entre ambos conocimientos. Por consiguiente, soslayar los postulados fundantes, los significados, los significantes e incluso las creencias presentes en los discursos de las disciplinas interactuantes no garantiza el cumplimiento de sus fines si necesita de ese otro conocimiento en la práctica disciplinal. Esta última afirmación debe ser acotada ya que la Tributación necesita en un mayor grado a la Contabilidad Financiera de lo que ella necesita de la Tributación. Como se dijo la Contabilidad Financiera es instrumental a la Tributación.

\subsection{EI lenguaje especializado y los modelos mentales}

Como último punto, cabe agregar la relación entre los modelos mentales y el lenguaje especializado. Los discursos se construyen e interpretan con base en modelos mentales que "son representaciones mentales subjetivas que hacen los actores sociales participantes de una situación comunicativa a partir de sus experiencias personales y sociales (Van Dijk, 2011 p. 14)" y esas representaciones mentales probablemente generen incompatibilidades comunicativas cuando son llevadas a estos discursos pues están soportadas por una diversidad de experiencias y creencias, adquiridas por el propio individuo, o construidas y reproducidas por sus comunidades.

Para este autor, las creencias son subsistemas que forman parte de un sistema más grande, el cual está integrado por otros componentes (contexto, cultura, genética, etc.) y al que denomina "modelos

1. Para profundizar sobre este debate, ver Hevia (2013) ARRENDAMIENTO FINANCIERO E IMPUESTO SOBRE LA RENTA, Una aproximación crítica desde la significación económica, Caracas, Venezuela p 150 en adelante.

REVISTA DE INVESTIGACIÓN SIGMA / Vol. 08, Nº 1, 2021 (pág. 59-70) 
mentales". Estos modelos permiten interpretar/ producir un determinado discurso en cualquier contexto social. Para este estudio, se asume que los modelos mentales de los practicantes de la disciplina contable y tributaria contienen no solo experiencias diferentes, sino creencias posiblemente encontradas vista las tensiones disciplinales descritas hasta el momento.

En los procesos de socialización, las personas "van adquiriendo nuevos conocimientos que se comparten con las comunidades epistémicas y que a posteriori influirán en la construcción de nuevos modelos mentales" (Van Dijk 2017 p. 3265) y con una lógica de lenguaje característica; es una cadena cognitiva infinita que hará que una determinada creencia sobreviva o no. En esta investigación, puede entonces sostenerse que los modelos mentales de las personas que producen/interpretan discursos contables y tributarios son los que definen qué es lo que se interpreta o qué es lo que se expresa en esos discursos, y que, vista la definición de modelo mental, tienen incorporados un sistema de creencias propio de los participantes de una acción comunicativa.

\subsection{El centro del debate}

Al igual que otros lenguajes especializados, la Contabilidad Financiera y la Tributación cuentan con un lenguaje propio cuyas palabras y signos intentan dar cuenta de la realidad económica y de las transacciones de las entidades, el cual permite interpretar esa realidad "a la que no se tiene acceso de otra forma" (Chua, 1995 p. 141) sino con ese lenguaje especializado. Pero, en criterio del investigador, se debe aclarar la función de la Contabilidad y distinguirla de la Contabilidad Financiera ya que la realidad económica que ambas pretenden representar es diferente, la primera mira al pasado, y la segunda mira hacia el futuro, por lo tanto, un lenguaje es prospectivo y el otro retrospectivo. El investigador sugiere que el abandono casi total de la economía por sobre las finanzas para la construcción del lenguaje que produce la información financiera es la que causa la mayoría de las tensiones entre la Contabilidad Financiera y la Tributación.
La Contabilidad intenta aprehender o capturar la mayor cantidad de rasgos una realidad que pasó, pero, parafraseando a Li (1963), no todos los eventos de la empírea económica pueden ser descritos por el lenguaje contable, por eso hace tanto énfasis en los "objetos, los precios, las unidades monetarias, las entradas y salidas, los periodos en que se producen las transacciones, los valores históricos y de adquisición," (Ordoñez et al 2008 p. 240) para intentar mitigar esas deficiencias propias que tienen los procesos de representación. Sobre estos referentes que se acaban de mencionar, y sus continuos cambios, se construyó su lenguaje.

La contabilidad realiza abstracciones graduales de la empírea económica y las expresa utilizando las reglas de designación que se originan a partir de sus propias definiciones, por lo que asumir que el lenguaje contable expresa totalmente esa realidad es una ingenuidad, dada las limitaciones propias del lenguaje y las posibles restricciones en el acceso a la información de la realidad que tengan los preparadores de información financiera y contable, pero hay algo innegable, su temporalidad es hacia el pasado al igual que lo hace la Tributación.

Es claro que al hacer aproximaciones de esos hechos económicos y transacciones se hace obligatorio comprender los significantes y significados que codifican y representan esas aproximaciones ya que, en su continua evolución, darán origen a la especialización del lenguaje contable. En ocasiones esta evolución es considerada por los productores del discurso contable cuando en estos se plasman los cambios que se producen en el contexto económico, en los agentes económicos y en la significación que sobre esos cambios le atribuye su propia comunidad epistémica.

No obstante, hay dos cosas que hay que destacar en esta especialización del lenguaje contable. La primera es que "la significación se hace obviando al lenguaje común" (Hevia 2012 p. 140) y la segunda, es que a pesar de que el núcleo de la Contabilidad se ha ido moviendo, en nuestro criterio la misma entró en un parálisis paradigmática a partir del hecho que el principal emisor de normas contables en el mundo sustituyó de facto a la Contabilidad por 
la Contabilidad Financiera como se explanó en la tensiones anterior (Ver posturas del autor sobre la razonabilidad de la información financiera, Revista Sigma, 2020). Esto causa que en la doctrina contable se confundan estos conocimientos y que se hagan grandes disertaciones asumiendo que se escribe sobre Contabilidad (histórica) cuando en realidad se escribe sobre Contabilidad Financiera (predictiva), y esto, afecta la relación interdisciplinal con la Tributación. Sobre contabilidad se volverá a escribir cuando se asuma la diferencia teleológica entre estos dos conocimientos y cada país asuma normas propias contables que tengan una visión temporal orientada al pasado.

\section{Conclusión}

Las palabras, sintagmas, cláusulas, enunciados, conceptos, principios presentes en los discursos contables y tributarios son tanto, una representación propia y específica de una realidad vivida por los practicantes que producen/interpretan estos discursos, como una representación de un conocimiento compartido socialmente por su respectiva comunidad epistémica, es decir, son una representación de sus modelos mentales.

Estas expresiones lingüísticas estarán construidas por su propia evaluación del mundo, sus emociones, sus creencias, sus intereses, su propia ontología, su cultura, por lo que es difícil pensar que disciplinas con objetivos, ideologías e intereses tan diferentes tengan discursos perfectamente compatibles, y menos desde la construcción de su lenguaje. No obstante, la praxis disciplinal demuestra que su interacción es continua y las obliga a comunicarse a través de sus respectivos discursos. Se demostró que, dada la temporalidad de ambos conocimientos, pudieran existir tensiones disciplinales que causen problemas en el ejercicio profesional del Contador Público.

En resumen, todo lo descrito hasta ahora sugeriría un alto grado de especialización en el lenguaje de ambas disciplinas y un grado de incompatibilidad en las estructuras semánticas y semióticas presentes en los discursos de ambos conocimientos, derivados posiblemente de sistemas de creencias disímiles.
Esto tiene consecuencias en la práctica disciplinal, pues como se afirmó, a mayor especialización del lenguaje disciplinal, menor las posibilidades de generar una comunicación efectivas entre ambos conocimientos.

\section{Referencias bibliográficas}

American Accounting Asociación (1966). A Statement of Basic Accounting Theory (ASOBAT). American Accounting Association Publications. Estados Unidos de América.

Arreghini, H. (2012). Contabilidad: Sus fundamentos conceptuales. Resumen de tesis doctoral. Argentina: Universidad de Buenos Aires. Disponible en http://ojs.uba.ar

Arthur Andersen \& Co. (1972). Objetivos de los Estados Financieros para las Empresas de Negocios. Arthur Andersen \& Co.

Arthur Andersen \& Co. (1974). Normas de Contabilidad para Empresas de Negocios en todo el mundo. Arthur Andersen \& Co.

Baudrillard, J. (1978). Cultura y Simulacros. Barcelona, España: Editorial Kairos.

Baudrillard, J. (1998). Simulacros y simulaciones. Estados Unidos: Stanford University Press.

Belkaoui, A. (1993). Accounting Theory. Hartcourt Brace, Jovanovich College Publishers, Gran Bretaña.

Berger, P. y Luckmann, T. (2001). La construcción social de la realidad. Argentina: Amorrotur Editores (décimo séptima impresión).

Biondi, Y., Glover, J., Jamal, K., Ohlson, J., Penman, S., Sunder, S. y Tsujiyama, E. (2012). Some Conceptual Tensions in Financial Reporting. Estados Unidos de América. Accounting Horinzons. Volumen 26. Número 1.pp. 125-133. Disponible en http://aaajournals.org

Boter, F. (1959). Las doctrinas contables. Editorial Juventud, S.A., Barcelona, España. Burton, J. (1976). Conflicts and compromises in financial 
reporting. Disponible en www.sechistorical.org

Cañibano, L., Tua, J., López, J. (1985). Naturaleza y filosofía de los principios contables. Revista Española de Financiación y Contabilidad. Volumen XV, número 47, pp. 293-355.

Cañibano, L. (1975). Teoría de la Contabilidad. Ed. ICE, Madrid, España.

Day, R. (2000). UK accounting regulation: an historical perspective. School of Finance \& Law Working Paper Series. Número 20. Bournemouth University.

Demkis, J., Fellingham, J., Ijiri, Y. y Sunder, S. (2002). Some thoughts on intellectual foundations of accounting. Conferencia de la Universidad Carnegie Mellon. Publicado en American Acoounting Association: Acounting Horizons. Volumen 16, número 2.pp. 157-168. Disponible en www.citeseerx.ist.psu.edu

Diccionario de la Real Academia de la Lengua Española. Versión electrónica (2020). Disponible en http://www.rae.es/recursos/ diccionarios/drae

Durán y Mejías (2013). La interdisciplina tributaria. Lima, Perú.

Dussel, E. (2000). Sobre el Sujeto y la Intersubjetividad; El Agente Histórico como Actor en los Movimientos Sociales. Disponible en www.Pasos841.htm (búsqueda realizada en 2018).

Financial Accounting Standards Board (FASB) and the International Accounting Standards Board (IASB) (2006). A Roadmap for Convergence between IFRSs and US GAAP- 2006-2008. Memorandum of Undrestanding between the FASB and the IASB. Statement. Disponible en www.ifrs.org

Financial Accounting Standards Board (FASB) and the International Accounting Standards Board (IASB) (2018). Preliminary Views, Conceptual Framework for Financial Reporting. Disponible en www.ifrs.org .
Ferullo, C.; Grecchi, A. y Vitta, J. (2000). La contabilidad como base de la determinación tributaria. Quintas Jornadas Investigaciones en la Facultad de Ciencias Económicas y Estadística. Universidad Nacional de Rosario. Noviembre. Argentina.

Hevia B. (2013) Arrendamiento Financiero E Impuesto Sobre La Renta. Una aproximación crítica desde la significación económica, Caracas, Venezuela

Pacheco, J (2013) Los conflictos en Contabilidad a luz del modelo de regulación contable internacional. Tesis doctoral. Mérida, Venezuela.

Romero Mucci, H (2009). El Derecho y el Revés de la Contabilidad. Caracas, Venezuela.

Searle J. (1997) La construcción social de la realidad. México. Paídos.

Saussure F (1915) Curso de lingüística general. París, Francia.

Van Dijk T. (2011) Sociedad y Discurso. Barcelona. España.

Bastidas Canelones, Jorge

Tensiones disciplinales entre la Contabilidad Financiera y la Tributación producidas por su lenguaje especializado 